Journal of Universal Mathematics

Vol.2 No.2 Pp.154-165 (2019)

ISSN-2618-5660

\title{
THEORY OF FRACTIONAL IMPLICIT DIFFERENTIAL EQUATIONS WITH COMPLEX ORDER
}

\author{
D. VIVEK, E. M. ELSAYED, AND K. KANAGARAJAN
}

\begin{abstract}
AвSTRACT. In this paper, we consider boundary value problems for the following nonlinear implicit differential equations with complex order

$$
\left\{\begin{array}{l}
D_{0^{+}}^{\theta} x(t)=f\left(t, x(t), D_{0^{+}}^{\theta} x(t)\right), \quad \theta=m+i \alpha, \quad t \in J:=[0, T], \\
a x(0)+b x(T)=c,
\end{array}\right.
$$

where $D_{0^{+}}^{\theta}$ is the Caputo fractional derivative of order $\theta \in \mathbb{C}$. Let $\alpha \in \mathbb{R}^{+}$, $0<\alpha<1, m \in(0,1]$, and $f: J \times \mathbb{R}^{2} \rightarrow \mathbb{R}$ is given continuous function. Here $a, b, c$ are real constants with $a+b \neq 0$.

We derive the existence and stability of solution for a class of boundary value problem(BVP) for nonlinear fractional implicit differential equations(FIDEs) with complex order. The results are based upon the Banach contraction principle and Schaefer's fixed point theorem.
\end{abstract}

\section{INTRODUCTION}

Fractional calculus(FC) is a generalization of ordinary differentiation and integration to arbitrary non-integer order. The subject is as old as the differential calculus, and goes back to the time when Leibnitz and Newton invented differential calculus. The idea of FC has been a subject of interest not only among mathematicians, but also among physicists and engineers. FC appears in rheology, viscoelasticity, electrochemistry, electromagnetism, etc. (see, for example, the books $[15,23,24,27]$ and references therein). However, most of the work done in this field so far has been based on the use of real order fractional derivatives and integrals. It is worth to mention that there are several authors who also applied complex order fractional derivative. Fractional operators of complex order are investigated as follows (see [21, 30]). In 1977, Ross Bertram [5] considered a use for a derivative of complex order in the fractional calculus. In [13], Carla M.A.Pinto studied a complex order van der Pol oscillator. Later, R. Andriambololona et al. [2] proposed some definitions of complex order integrals and complex order derivatives using operator approach. For instance, some basic theory for fractional differential equations(FDEs) with complex order was investigated by Neamaty et al.[26]. They derived sufficient conditions for existence of solutions of fractional boundary value problems with complex order. Recently, Teodor M.

2000 Mathematics Subject Classification. 26A33.

Key words and phrases. Implicit differential equation; Boundary value problem; Stirling asymptotic formula; fractional derivative; Existence; Ulam stability. 
Atanackovi et al. established complex order fractional derivatives in models that describe viscoelastic materials in [31]. The authors investigated existence and uniqueness by using a classical fixed point theorem.

BVPs for FDEs have been considered by some authors (see $[4,6,7,8,9,25,36]$ and references therein).

In the theory of functional equations there are some special kind of data dependence: see, for example, Jung[19], Rus[29], S. M. Ulam[32] and we refer to $[1,16,17]$. In recent years, many people have paid more and more attention to Hyers-Ulam stability of differential equations, and gained a series of results. However, the theory of Hyers-Ulam stability of FDEs is still in the initial stages. There are some papers $[3,18,22,28,34,35]$ treating with Ulam-Hyers stability for FDEs. The investigation of Hyers-Ulam stability of FDEs with complex order started recently and we should mention here the results obtained on this direction by [33].

The existence of solutions of this kind of BVP has been studied by Benchohra et al., for example, in [10, 11, 12].

The rest of this paper is organized as follows. In Section 2, we give some notations; recall some concepts and preparation results. In Section 3, we give existence and uniqueness results of solutions for the problem (0.1) by Schaefer's fixed point theorem. In Section 4, We present the Ulam stability results for the problem (0.1).

\section{Preliminaries on COMPLeX ORder}

We first wish to collect some basic lemmas that will be important to us in what follows. For further reading and details on complex order in FC, we refer to the papers, for example, in [21, 30,31].

By $C(J, \mathbb{R})$ we denote the Banach space of all continuous functions $J$ into $\mathbb{R}$ with the norm

$$
\|x\|_{\infty}:=\sup \{|x(t)|: t \in J\} .
$$

By $L^{1}(J)$ we denote the space of Lebesgue-integrable function $x: J \rightarrow \mathbb{R}$ with the norm $\|x\|_{L^{1}}=\int_{0}^{T}|x(t)| d t$.

Definition 2.1. ([27]) The Riemann-Liouville fractional integral of order $v \in \mathbb{C}$, $(\operatorname{Re}(v)>0)$ of a function $f:(0, \infty) \rightarrow \mathbb{R}$ is

$$
I_{0^{+}}^{v} f(t)=\frac{1}{\Gamma(v)} \int_{0}^{t}(t-s)^{v-1} f(s) d s .
$$

Definition 2.2. ([27]) For a function $f$ given by on the interval $J$, the Caputo fractional-order $v \in \mathbb{C},(\operatorname{Re}(v)>0)$ of $f$, is defined by

$$
\left(D_{0^{+}}^{v} f\right)(t)=\frac{1}{\Gamma(n-v)} \int_{0}^{t}(t-s)^{n-v-1} f^{(n)}(s) d s,
$$

where $n=[\operatorname{Re}(v)]+1$ and $[\operatorname{Re}(v)]$ denotes the integral part of the real number $v$.

Definition 2.3. ([20]) The Stirling asymptotic formula of the Gamma function for $z \in \mathbb{C}$ is following

$$
\Gamma(z)=(2 \pi)^{\frac{1}{2}} z^{\frac{z-1}{2}} e^{-z}\left[1+O\left(\frac{1}{z}\right)\right], \quad(|\arg (z)|<\pi ;|z| \rightarrow \infty),
$$


and its results for $|\Gamma(u+i v)|,(u, v \in \mathbb{R})$ is

$$
|\Gamma(u+i v)|=(2 \pi)^{\frac{1}{2}}|v|^{u-\frac{1}{2}} e^{-u-\pi|v| / 2}\left[1+O\left(\frac{1}{v}\right)\right], \quad(v \rightarrow \infty) .
$$

Theorem 2.4. ([14])(Banach's fixed point theorem). Let $C$ be a non-empty closed subset of a Banach space $X$, then any contraction mapping $T$ of $C$ into itself has a unique fixed point.

Theorem 2.5. ([14])(Schaefer's fixed point theorem). Let $P: C(J, \mathbb{R}) \rightarrow C(J, \mathbb{R})$ completely continuous operator. If the set

$$
\zeta=\{x \in C(J, \mathbb{R}): x=\delta(P x) \text { for some } \delta \in[0, T]\}
$$

is bounded, then $P$ has at least a fixed point.

\section{EXISTENCE AND UNIQUENESS RESULTS}

Let us start by defining what we mean by a solution of the problem (0.1). We adopt some ideas from [12].

Definition 3.1. A function $x \in C(J, \mathbb{R})$ is said to be a solution of (0.1) if $x$ satisfies the equation $D_{0^{+}}^{\theta} x(t)=f\left(t, x(t), D_{0^{+}}^{\theta} x(t)\right)$ on $J$, and the condition $a x(0)+$ $b x(T)=c$.

For the existence of solutions for the problem (0.1), we need the following auxiliary lemma.

Lemma 3.2. Let $\theta=m+i \alpha, 0<m \leq 1, \alpha \in \mathbb{R}^{+}$and $f: J \times \mathbb{R}^{2} \rightarrow \mathbb{R}$ be continuous. A function $x$ is a solution of the fractional integral equation

$$
x(t)=x_{0}+\frac{1}{\Gamma(\theta)} \int_{0}^{t}(t-s)^{\theta-1} f\left(s, x(s), D_{0^{+}}^{\theta} x(s)\right) d s
$$

if and only if $x$ is a solution of the initial value problem for the following FIDE with complex order

$$
\begin{aligned}
& D_{0^{+}}^{\theta} x(t)=f\left(t, x(t), D_{0^{+}}^{\theta} x(t)\right), \quad t \in J:=[0, T] \\
& x(0)=x_{0} .
\end{aligned}
$$

As a consequence of Lemma 3.2 we have the following result which is useful in what follows.

Lemma 3.3. Let $\theta=m+i \alpha, 0<m \leq 1, \alpha \in \mathbb{R}^{+}$and $f: J \times \mathbb{R}^{2} \rightarrow \mathbb{R}$ be continuous. A function $x$ is a solution of the fractional integral equation

$$
\begin{aligned}
x(t)= & \frac{1}{\Gamma(\theta)} \int_{0}^{t}(t-s)^{\theta-1} f\left(s, x(s), D_{0^{+}}^{\theta} x(s)\right) d s \\
& -\frac{1}{a+b}\left[\frac{b}{\Gamma(\theta)} \int_{0}^{T}(T-s)^{\theta-1} f\left(s, x(s), D_{0^{+}}^{\theta} x(s)\right) d s-c\right]
\end{aligned}
$$

if and only if $x$ is a solution of the BVP for FIDEs with complex order

$$
\begin{aligned}
& D_{0^{+}}^{\theta} x(t)=f\left(t, x(t), D_{0^{+}}^{\theta} x(t)\right), \quad t \in[0, T], \\
& a x(0)+b x(T)=c .
\end{aligned}
$$

Our first result is based on the Banach contraction principle. 
Theorem 3.4. Assume that the following hypotheses are fulfilled.

(H1) $f: J \times \mathbb{R}^{2} \rightarrow \mathbb{R}$ is continuous function.

(H2) There exist constants $K>0$ and $L>0$ such that

$$
|f(t, u, v)-f(t, \bar{u}, \bar{v})| \leq K|u-\bar{u}|+L|v-\bar{v}|,
$$

for any $u, v, \bar{u}, \bar{v} \in \mathbb{R}$ and $t \in J$.

If $\Omega_{K, L, m, T, a, b, \theta}<1$, then the problem (0.1) has a unique solution on $J$.

Proof. Transform the problem (0.1) into a fixed point problem.

Consider the operator $P: C(J, \mathbb{R}) \rightarrow C(J, \mathbb{R})$ defined by

$$
(P x)(t)=\frac{1}{\Gamma(\theta)} \int_{0}^{t}(t-s)^{\theta-1} K_{x}(s) d s-\frac{1}{a+b}\left[\frac{b}{\Gamma(\theta)} \int_{0}^{T}(T-s)^{\theta-1} K_{x}(s) d s-c\right] .
$$

For sake of brevity, let us take

$$
\begin{aligned}
K_{x}(t) & =D_{0^{+}}^{\theta} x(t) \\
& =f\left(t, x(t), D_{0^{+}}^{\theta} x(t)\right) \\
& =f\left(t, x(t), K_{x}(t)\right) .
\end{aligned}
$$

Clearly, the fixed points of the operator $P$ are solutions of the problem (0.1). We shall use the Banach contraction principle to prove that $P$ defined by (3.7) has a fixed point. We shall show that $P$ is a contraction.

Let $x, y \in C(J, \mathbb{R})$. Then, for each $t \in J$ we have

$$
\begin{aligned}
|(P x)(t)-(P y)(t)| \leq & \frac{1}{|\Gamma(\theta)|} \int_{0}^{t}\left|(t-s)^{\theta-1}\right|\left|K_{x}(s)-K_{y}(s)\right| d s \\
& +\frac{|b|}{|\Gamma(\theta)||a+b|} \int_{0}^{T}\left|(T-s)^{\theta-1}\right|\left|K_{x}(s)-K_{y}(s)\right| d s .
\end{aligned}
$$

Here

$$
\begin{aligned}
\left|K_{x}(t)-K_{y}(t)\right| & \leq\left|f\left(t, x(t), K_{x}(t)\right)-f\left(t, y(t), K_{y}(t)\right)\right| \\
& \leq K|x(t)-y(t)|+L\left|K_{x}(t)-K_{y}(t)\right| \\
& \leq\left(\frac{K}{1-L}\right)|x(t)-y(t)| .
\end{aligned}
$$

By replacing eqn.(3.9) in the inequality eqn.(3.8), we obtain

$$
\begin{aligned}
& |(P x)(t)-(P y)(t)| \\
\leq & \left(\frac{K}{1-L}\right) \frac{1}{|\Gamma(\theta)|} \int_{0}^{t}\left|(t-s)^{\theta-1}\right||x(s)-y(s)| d s \\
& +\left(\frac{K}{1-L}\right) \frac{|b|}{|a+b|} \frac{1}{|a+b|} \frac{1}{|\Gamma(\theta)|} \int_{0}^{T}\left|(T-s)^{\theta-1}\right| \mid x(s)-y(s \mid d s \\
\leq & \left(\frac{K}{1-L}\right) \frac{1}{|\Gamma(\theta)|}\|x-y\|_{\infty} \int_{0}^{t}(t-s)^{m-1} d s+\left(\frac{K}{1-L}\right) \frac{|b|}{|a+b|} \frac{\|x-y\|_{\infty}}{|\Gamma(\theta)|} \int_{0}^{T}(T-s)^{m-1} d s \\
\leq & \left(\frac{K}{1-L} \frac{T^{m}}{m|\Gamma(\theta)|}\left[1+\frac{|b|}{|a+b|}\right]\right)\|x-y\|_{\infty} .
\end{aligned}
$$

Thus

$$
\|P x-P y\|_{\infty} \leq \Omega_{K, L, m, T, a, b, \theta}\|x-y\|_{\infty}
$$


where

$$
\Omega_{K, L, m, T, a, b, \theta}:=\left(\frac{K}{1-L} \frac{T^{m}}{m|\Gamma(\theta)|}\left[1+\frac{|b|}{|a+b|}\right]\right),
$$

depends only on the parameters of the problem. And since $\Omega_{K, L, m, T, a, b, \theta}<1$, the results follows in view of the contraction mapping principle.

We now can prove the following existence result.

Theorem 3.5. Under the hypotheses of Theorem 3.4 and

(H3) There exist $l, p, q \in C(J, \mathbb{R})$ with $l^{*}=\sup _{t \in J} l(t)<t$ such that

$$
|f(t, u, v)| \leq l(t)+p(t)|u|+q(t)|v|,
$$

for $t \in J, u, v \in \mathbb{R}$,

hold. Then the problem (0.1) has at least one solution on J.

Proof. We shall use Schaefer's fixed point theorem to prove that $P$ defined by (3.7) has a fixed point. The proof will be given in several steps.

Claim 1: $P$ is continuous.

Let $\left\{x_{n}\right\}$ be a sequence such that $x_{n} \rightarrow x$ in $C(J, \mathbb{R})$. Then for each $t \in J$

$$
\begin{aligned}
\mid & \left(P x_{n}\right)-(P x)(t) \mid \\
\leq & \frac{1}{|\Gamma(\theta)|} \int_{0}^{t}\left|(t-s)^{\theta-1}\right|\left|K_{x_{n}}(s)-K_{x}(s)\right| d s \\
& +\frac{|b|}{|\Gamma(\theta)||a+b|} \int_{0}^{T}\left|(T-s)^{\theta-1}\right|\left|K_{x_{n}}(s)-K_{x}(s)\right| d s \\
\leq & \frac{1}{|\Gamma(\theta)|} \int_{0}^{t}\left|(t-s)^{\theta-1}\right| \sup _{s \in J}\left|K_{x_{n}}(s)-K_{x}(s)\right| d s+\frac{|b|}{|\Gamma(\theta)||a+b|} \int_{0}^{T}\left|(T-s)^{\theta-1}\right| \sup _{s \in J}\left|K_{x_{n}}(s)-K_{x}(s)\right| d s \\
\leq & \frac{\left\|K_{x_{n}}(\cdot)-K_{x}(\cdot)\right\|_{\infty}}{|\Gamma(\theta)|}\left[\int_{0}^{t}(t-s)^{m-1} d s+\frac{|b|}{|a+b|} \int_{0}^{T}(T-s)^{m-1} d s\right] \\
\leq & \frac{T^{m}\left\|K_{x_{n}}(\cdot)-K_{x}(\cdot)\right\|_{\infty}}{m|\Gamma(\theta)|}\left(1+\frac{|b|}{|a+b|}\right) .
\end{aligned}
$$

Since $f$ is a continuous function, we have

$$
\left\|P x_{n}-P x\right\|_{\infty} \rightarrow 0 \text { as } n \rightarrow \infty .
$$

Claim 2: $P$ maps bounded sets into bounded sets in $C(J, \mathbb{R})$.

Indeed, it is enough to show that for any $\eta^{*}>0$, there exists a positive constant $\chi$ such that for each $x \in B_{\eta^{*}}=\left\{x \in C(J, \mathbb{R}):\|x\|_{\infty} \leq \eta^{*}\right\}$, we have $\|P x\|_{\infty} \leq \chi$.

$$
\begin{aligned}
|(P x)(t)| \leq & \frac{1}{|\Gamma(\theta)|} \int_{0}^{t}\left|(t-s)^{\theta-1}\right|\left|K_{x}(s)\right| d s \\
& \frac{|b|}{|a+b|} \int_{0}^{T}\left|(T-s)^{\theta-1}\right|\left|K_{x}(s)\right| d s+\frac{|c|}{|a+b|},
\end{aligned}
$$


and by $(\mathrm{H} 3)$, we have

$$
\begin{aligned}
\left|K_{x}(t)\right| & \leq\left|f\left(t, x(t) K_{x}(t)\right)\right| \\
& \leq l(t)+p(t)|x(t)|+q(t)\left|K_{x}(t)\right| \\
& \leq l^{*}+p^{*}|x(t)|+q^{*}\left|K_{x}(t)\right| \\
& \leq \frac{l^{*}+p^{*}|x(t)|}{1-q^{*}} .
\end{aligned}
$$

By replacing eqn. (3.11) in the inequality (3.10), we get

$$
\begin{aligned}
|(P x)(t)| \leq & \frac{1}{|\Gamma(\theta)|} \int_{0}^{t}\left|(t-s)^{\theta-1}\right|\left(\frac{l^{*}+p^{*}|x(s)|}{1-q^{*}}\right) d s \\
& +\frac{|b|}{|a+b||\Gamma(\theta)|} \int_{0}^{T}\left|(T-s)^{\theta-1}\right|\left(\frac{l^{*}+p^{*}|x(s)|}{1-q^{*}}\right) d s+\frac{|c|}{|a+b|} . \\
:= & A_{1}+A_{2} . \\
A_{1}= & \frac{1}{|\Gamma(\theta)|} \int_{0}^{t}\left|(t-s)^{\theta-1}\right|\left(\frac{l^{*}+p^{*}|x(s)|}{1-q^{*}}\right) d s \\
= & \frac{T^{m}}{\left(1-q^{*}\right)|\Gamma(\theta)|}\left(\frac{l^{*}}{m}+\frac{p^{*}|| x \|_{\infty}}{m}\right) . \\
A_{2}= & \frac{|a+b||b|}{|\Gamma(\theta)|} \int_{0}^{T}\left|(T-s)^{\theta-1}\right|\left(\frac{l^{*}+p^{*}|x(s)|}{1-q^{*}}\right) d s \\
= & \frac{|b| T^{m}}{\left(1-q^{*}\right) m|\Gamma(\theta)||a+b|}\left(l^{*}+p^{*}\|x\|_{\infty}\right) .
\end{aligned}
$$

To substitute $A_{1}, A_{2}$ values into eqn. (3.12),we have

$$
\begin{aligned}
|(P x)(t)| \leq & \frac{T^{m} l^{*}}{\left(1-q^{*}\right)} \frac{1}{m|\Gamma(\theta)|}\left(1+\frac{|b|}{|a+b|}\right) \\
& +\frac{T^{m} p^{*}}{\left(1-q^{*}\right)} \frac{1}{m|\Gamma(\theta)|}\left(1+\frac{|b|}{|a+b|}\right)\|x\|_{\infty}+\frac{|c|}{|a+b|} \\
:= & \chi
\end{aligned}
$$

Claim 3: $P$ maps bounded sets into equicontinuous sets of $C(J, \mathbb{R})$.

Let $t_{1}, t_{2} \in[0, T], t_{1}<t_{2}, B_{\eta^{*}}$ be a bounded set of $C(J, \mathbb{R})$ as in above steps, and let $x \in B_{\eta^{*}}$. Using the fact $f$ is bounded on the compact set $J \times B_{\eta^{*}}$ (thus $\left.\sup _{(t, x) \in J \times B_{\eta^{*}}}\left\|K_{x}(t)\right\|:=C_{0}<\infty\right)$. We will get

$$
\begin{aligned}
& \left|(P x)\left(t_{2}\right)-(P x)\left(t_{2}\right)\right| \\
& \leq\left|\frac{1}{\Gamma(\theta)} \int_{0}^{t_{1}}\left[\left(t_{2}-s\right)^{\theta-1}-\left(t_{1}-s\right)^{\theta-1}\right] K_{x}(s) d s+\frac{1}{\Gamma(\theta)} \int_{t_{1}}^{t_{2}}\left(t_{2}-s\right)^{\theta-1} K_{x}(s) d s\right| \\
& \leq \frac{C_{0}}{|\Gamma(\theta)|} \int_{0}^{t_{1}}\left|\left[\left(t_{1}-s\right)^{\theta-1}-\left(t_{2}-s\right)^{\theta-1}\right]\right| d s+\frac{C_{0}}{|\Gamma(\theta)|} \int_{t_{1}}^{t_{2}}\left|\left(t_{2}-s\right)^{\theta-1}\right| d s \\
& \leq \frac{C_{0}}{|\Gamma(\theta)|}\left|2\left(t_{1}-t_{2}\right)^{\theta}+t_{2}^{\theta}-t_{1}^{\theta}\right|
\end{aligned}
$$

As $t_{1} \rightarrow t_{2}$, the right-hand side of the above inequality tends to zero. As a consequence of previous discussion with the Arzela-Ascoli theorem, we can 
conclude that $P: C(J, \mathbb{R}) \rightarrow C(J, \mathbb{R})$ is continuous and completely continuous. Claim 4: A priori bounds.

Now it remains to show that the set

$$
\zeta=\{x \in C(J, \mathbb{R}) ; x=\delta(P x) \text { for some } 0<\delta<1\}
$$

is bounded. Let $x \in \zeta$, then $x=\delta(P x)$ for some $0<\delta<1$. Thus, for each $t \in J$, we have

$$
x(t)=\frac{\delta}{\Gamma(\theta)} \int_{0}^{t}(t-s)^{\theta-1} K_{x}(s) d s-\frac{\delta}{a+b}\left[\frac{b}{\Gamma(\theta)} \int_{0}^{T}(T-s)^{\theta-1} K_{x}(s) d s-c\right] .
$$

This implies by (H3) that for $t \in J$, we have

$$
\begin{aligned}
|(P x)(t)| \leq & \frac{T^{m} l^{*}}{\left(1-q^{*}\right)} \frac{1}{m|\Gamma(\theta)|}\left(1+\frac{|b|}{|a+b|}\right) \\
& +\frac{T^{m} p^{*}}{\left(1-q^{*}\right)} \frac{1}{m|\Gamma(\theta)|}\left(1+\frac{|b|}{|a+b|}\right)\|x\|_{\infty}+\frac{|c|}{|a+b|} .
\end{aligned}
$$

Thus for every $t \in J$, we have

$$
\begin{aligned}
\|P x\|_{\infty} \leq & \frac{T^{m} l^{*}}{\left(1-q^{*}\right)} \frac{1}{m|\Gamma(\theta)|}\left(1+\frac{|b|}{|a+b|}\right) \\
& +\frac{T^{m} p^{*}}{\left(1-q^{*}\right)} \frac{1}{m|\Gamma(\theta)|}\left(1+\frac{|b|}{|a+b|}\right)\|x\|_{\infty}+\frac{|c|}{|a+b|} \\
:= & R .
\end{aligned}
$$

This show that the set $\zeta$ is bounded. As a consequence of Schaefer's fixed point theorem, we deduce that $P$ has a fixed point which is a solution of the problem (0.1).

\section{Stability Results}

In this section, we consider the Ulam stability of nonlinear FIDEs (0.1). Now we consider the Ulam stability for the problem

$$
D_{0^{+}}^{\theta} x(t)=f\left(t, x(t), D_{0^{+}}^{\theta} x(t)\right), \quad \theta=m+i \alpha, t \in[0, T],
$$

and the following inequations:

$$
\begin{aligned}
& \left|D_{0^{+}}^{\theta} z(t)-f\left(t, z(t), D_{0^{+}}^{\theta} z(t)\right)\right| \leq \epsilon, t \in[0, T], \\
& \left|D_{0^{+}}^{\theta} z(t)-f\left(t, z(t), D_{0^{+}}^{\theta} z(t)\right)\right| \leq \epsilon \varphi(t), t \in[0, T], \\
& \left|D_{0^{+}}^{\theta} z(t)-f\left(t, z(t), D_{0^{+}}^{\theta} z(t)\right)\right| \leq \varphi(t), t \in[0, T] .
\end{aligned}
$$

Definition 4.1. The equation (4.1) is Ulam-Hyers stable if there exists a real number $C_{f}>0$ such that for each $\epsilon>0$ and for each solution $z \in C(J, \mathbb{R})$ of the inequality (4.2) there exists a solution $x \in C(J, \mathbb{R})$ of equation (4.1) with

$$
|z(t)-x(t)| \leq C_{f} \epsilon, t \in J \text {. }
$$


Definition 4.2. The equation (4.1) is generalized Ulam-Hyers stable if there exists $\psi_{f} \in C([0, \infty),[0, \infty)), \psi_{f}(0)=0$ such that for each solution $z \in C(J, \mathbb{R})$ of the inequality (4.2) there exists a solution $x \in C(J, \mathbb{R})$ of equation (4.1) with

$$
|z(t)-x(t)| \leq \psi_{f} \epsilon, \quad t \in J .
$$

Definition 4.3. The equation (4.1) is Ulam-Hyers-Rassias stable with respect to $\varphi \in C(J, \mathbb{R})$ if there exists a real number $C_{f}>0$ such that for each $\epsilon>0$ and for each solution $z \in C(J, \mathbb{R})$ of the inequality (4.3) there exists a solution $x \in C(J, \mathbb{R})$ of equation (4.1) with

$$
|z(t)-x(t)| \leq C_{f} \epsilon \varphi(t), \quad t \in J .
$$

Definition 4.4. The equation (4.1) is generalized Ulam-Hyers-Rassias stable with respect to $\varphi \in C(J, \mathbb{R})$ if there exists a real number $C_{f, \varphi}>0$ such that for each solution $z \in C(J, \mathbb{R})$ of the inequality (4.4) there exists a solution $x \in C(J, \mathbb{R})$ of equation (4.1) with

$$
|z(t)-x(t)| \leq C_{f, \varphi} \varphi(t), \quad t \in J .
$$

Remark 4.5. A function $z \in C(J, \mathbb{R})$ is a solution of (4.2) if and only if there exists a function $g \in C(J, \mathbb{R})$ (which depend on $z$ ) such that

(1) $|g(t)| \leq \epsilon, t \in J$;

(2) $D_{0^{+}}^{\theta} z(t)=f\left(t, z(t), D_{0^{+}}^{\theta} z(t)\right)+g(t), t \in J$.

Remark 4.6. Let $\theta=m+i \alpha, m \in(0,1]$ and $\alpha \in \mathbb{R}^{+}$, if $z \in C(J, \mathbb{R})$ is a solution of the inequality

$$
\left|D_{0^{+}}^{\theta} z(t)-f\left(t, z(t), D_{0^{+}}^{\theta} z(t)\right)\right| \leq \epsilon, t \in J,
$$

then $z$ is a solution of the following integral inequality

$$
\left|z(t)-A_{z}-\frac{1}{\Gamma(\theta)} \int_{0}^{t}(t-s)^{\theta-1} f\left(s, z(s), D_{0^{+}}^{\theta} z(s)\right) d s\right| \leq \frac{\epsilon}{|\Gamma(\theta)|} \frac{T^{m}}{m}\left(1+\frac{|b|}{|a+b|}\right) .
$$

Indeed, by Remark 4.5, we have that

$$
D_{0^{+}}^{\theta} z(t)=f\left(t, z(t), D_{0^{+}}^{\theta} z(t)\right)+g(t), \quad t \in J .
$$

Then

$$
\begin{aligned}
z(t)= & A_{z}+\frac{1}{\Gamma(\theta)} \int_{0}^{t}(t-s)^{\theta-1} f\left(s, z(s), D_{0^{+}}^{\theta} z(s)\right) d s+\frac{1}{\Gamma(\theta)} \int_{0}^{t}(t-s)^{\theta-1} g(s) d s \\
& -\left(\frac{b}{a+b}\right) \frac{1}{\Gamma(\theta)} \int_{0}^{T}(T-s)^{\theta-1} g(s) d s, t \in J .
\end{aligned}
$$

with

$$
A_{z}=\frac{1}{a+b}\left[c-\frac{b}{\Gamma(\theta)} \int_{0}^{T}(T-s)^{\theta-1} f\left(s, z(s), D_{0^{+}}^{\theta} z(s)\right) d s\right]
$$


From this it follows that

$$
\begin{aligned}
& \left|z(t)-A_{z}-\frac{1}{\Gamma(\theta)} \int_{0}^{t}(t-s)^{\theta-1} f\left(s, z(s), D_{0^{+}}^{\theta} z(s)\right) d s\right| \\
& =\left|\frac{1}{\Gamma(\theta)} \int_{0}^{t}(t-s)^{\theta-1} g(s) d s-\left(\frac{b}{a+b}\right) \frac{1}{\Gamma(\theta)} \int_{0}^{T}(T-s)^{\theta-1} g(s) d s\right| \\
& \leq \frac{1}{|\Gamma(\theta)|} \int_{0}^{t}(t-s)^{m-1}|g(s)| d s+\left(\frac{|b|}{|a+b|}\right) \frac{1}{|\Gamma(\theta)|} \int_{0}^{T}(T-s)^{m-1}|g(s)| d s \\
& \leq \frac{\epsilon}{|\Gamma(\theta)|} \frac{T^{m}}{m}\left(1+\frac{|b|}{|a+b|}\right) .
\end{aligned}
$$

Remark 4.7. Clearly,

(1) Definition $4.1 \Rightarrow$ Definition 4.2.

(2) Definition $4.3 \Rightarrow$ Definition 4.4 .

Remark 4.8. A solution of the FIDEs with complex order inequality

$$
\left|D_{0^{+}}^{\theta} z(t)-f\left(t, z(t), D_{0^{+}}^{\theta} z(t)\right)\right| \leq \epsilon, \quad t \in J,
$$

is called an fractional $\epsilon$-solution of the problem (4.1).

Lemma 4.9. (see Lemma 7.1.1,([16])) Let $z, w:[0, T) \rightarrow[0, \infty)$ be continuous functions where $T \leq \infty$. If $w$ is nondecreasing and there are constants $k \geq 0$ and $0<v<1$ such that

then

$$
z(t) \leq w(t)+k \int_{0}^{t}(t-s)^{v-1} z(s) d s, \quad t \in[0, T),
$$

$$
z(t) \leq w(t)+\int_{0}^{t}\left(\sum_{n=1}^{\infty} \frac{(k \Gamma(v))^{n}}{\Gamma(n v)}(t-s)^{n v-1} w(s)\right) d s, \quad t \in[0, T) .
$$

Remark 4.10. Under the hypothesis of Lemma 4.9, let $w(t)$ be a nondecreasing function on $[0, T)$. Then we have $z(t) \leq w(t) E_{v, 1}\left(k \Gamma(v) t^{v}\right)$.

Theorem 4.11. Assume that (H1),(H2) and $\Omega_{K, L, m, T, a, b, \theta}<1$ are fulfilled. Then, the problem (0.1) is Ulam-Hyers stable.

Proof. Let $\epsilon>0$ and let $z \in C(J, \mathbb{R})$ be a function which satisfies the inequality

$$
\left|D_{0^{+}}^{\theta} z(t)-f\left(t, z(t), D_{0^{+}}^{\theta} z(t)\right)\right| \leq \epsilon, \quad \text { for some, } \quad t \in J,
$$

and let $x \in C(J, \mathbb{R})$ be the unique solution of the following problem

$$
\begin{aligned}
& D_{0^{+}}^{\theta} x(t)=f\left(t, x(t), D_{0^{+}}^{\theta} x(t)\right), \quad t \in J, \quad \theta=m+i \alpha, \\
& x(0)=z(0), \quad x(T)=z(T),
\end{aligned}
$$

where $m \in(0,1]$ and $\alpha \in \mathbb{R}^{+}$.

Using Lemma 3.3, we obtain

$$
x(t)=A_{x}+\frac{1}{\Gamma(\theta)} \int_{0}^{t}(t-s)^{\theta-1} K_{x}(s) d s
$$

with

$$
A_{x}=\frac{1}{a+b}\left[c-\frac{b}{\Gamma(\theta)} \int_{0}^{T}(t-s)^{\theta-1} K_{x}(s) d s\right] .
$$


On the other hand, if $x(T)=z(T)$ and $x(0)=z(0)$, then $A_{x}=A_{z}$.

Indeed,

$$
\begin{aligned}
\left|A_{x}-A_{z}\right| & \leq \frac{|b|}{|a+b||\Gamma(\theta)|} \int_{0}^{T}\left|(T-s)^{\theta-1}\right|\left|K_{x}(s)-K_{z}(s)\right| d s \\
& \leq\left(\frac{K}{1-L}\right) \frac{|b|}{|a+b|} I_{0^{+}}^{\theta}|x(T)-z(T)| \\
& =0 .
\end{aligned}
$$

Thus, $A_{x}=A_{z}$. We have

$$
x(t)=A_{z}+\frac{1}{\Gamma(\theta)} \int_{0}^{t}(t-s)^{\theta-1} K_{x}(s) d s .
$$

By integration of the inequality (4.5) and using Remark 4.6, we obtain

$$
\left|z(t)-A_{z}-\frac{1}{\Gamma(\theta)} \int_{0}^{t}(t-s)^{\theta-1} K_{z}(s) d s\right| \leq \frac{\epsilon T^{m}}{m|\Gamma(\theta)|}\left(1+\frac{|b|}{|a+b|}\right) .
$$

We have for any $t \in J$

$$
\begin{aligned}
|z(t)-x(t)| \leq & \left|z(t)-A_{z}-\frac{1}{\Gamma(\theta)} \int_{0}^{t}(t-s)^{\theta-1} K_{z}(s) d s\right| \\
& +\frac{1}{|\Gamma(\theta)|} \int_{0}^{t}\left|(t-s)^{\theta-1}\right|\left|K_{z}(s)-K_{x}(s)\right| d s \\
\leq & \frac{\epsilon T^{m}}{m|\Gamma(\theta)|}\left(1+\frac{|b|}{|a+b|}\right)+\left(\frac{K}{1-L}\right) \frac{1}{|\Gamma(\theta)|} \int_{0}^{t}(t-s)^{m-1}|z(s)-x(s)| d s .
\end{aligned}
$$

Using Gronwall inequality, Lemma 4.9 and Remark 4.10, we obtain

$$
|z(t)-x(t)| \leq\left(1+\frac{|b|}{|a+b|}\right) \frac{\epsilon T^{m}}{m|\Gamma(\theta)|} E_{m, 1}\left(\frac{K}{1-L} \frac{1}{|\Gamma(\theta)|} \Gamma(m) T^{m}\right) .
$$

Thus, the problem (0.1) is Ulam-Hyers stable.

In the sequel we use of the following hypothesis.

(H4) There exists an increasing function $\varphi \in C(J, \mathbb{R})$ and there exists $\lambda_{\varphi}>0$ such that for any $t \in J$

$$
I_{0^{+}}^{\theta} \varphi(t) \leq \lambda_{\varphi} \varphi(t)
$$

Theorem 4.12. Assume that (H1),(H2),(H4) and $\Omega_{K, L, m, T, a, b, \theta}<1$ are fulfilled. Then, the problem (0.1) is generalized Ulam-Hyers-Rassias stable.

Proof. Let $z \in C(J, \mathbb{R})$ be solution of the following inequality

$$
\left|D_{0^{+}}^{\theta} z(t)-f\left(t, z(t), D_{0^{+}}^{\theta} z(t)\right)\right| \leq \epsilon \varphi(t), \quad t \in J, \quad \epsilon>0,
$$

and let $x \in C(J, \mathbb{R})$ be the unique solution of the following problem

$$
\begin{aligned}
& D_{0^{+}}^{\theta} x(t)=f\left(t, x(t), D_{0^{+}}^{\theta} x(t)\right), \quad t \in[0, T], \quad \theta=m+i \alpha, \\
& x(0)=z(0), \quad x(T)=z(T),
\end{aligned}
$$

where $m \in(0,1]$ and $\alpha \in \mathbb{R}^{+}$.

By Lemma 3.3, we get

$$
x(t)=A_{z}+\frac{1}{\Gamma(\theta)} \int_{0}^{t}(t-s)^{\theta-1} K_{x}(s) d s
$$


with

$$
A_{z}=\frac{1}{a+b}\left[c-\frac{b}{\Gamma(\theta)} \int_{0}^{T}(T-s)^{\theta-1} K_{z}(s) d s\right] .
$$

By integration of the inequality (4.6) and using (H4), we obtain

$$
\left|z(t)-A_{z}-\frac{1}{\Gamma(\theta)} \int_{0}^{t}(t-s)^{\theta-1} K_{z}(s) d s\right| \leq \epsilon \lambda_{\varphi} \varphi(t)\left(1+\frac{|b|}{|a+b|}\right) .
$$

We have for any $t \in J$

$$
\begin{aligned}
& |z(t)-x(t)| \\
& \leq\left|z(t)-A_{z}-\frac{1}{\Gamma(\theta)} \int_{0}^{t}(t-s)^{\theta-1} K_{z} d s\right|+\frac{1}{|\Gamma(\theta)|} \int_{0}^{t}\left|(t-s)^{\theta-1}\right|\left|K_{z}(s)-K_{x}(s)\right| d s \\
& \leq\left(1+\frac{|b|}{|a+b|}\right) \epsilon \lambda_{\varphi} \varphi(t)+\left(\frac{K}{1-L}\right) \frac{1}{|\Gamma(\theta)|} \int_{0}^{t}(t-s)^{m-1}|z(s)-x(s)| d s .
\end{aligned}
$$

Using Gronwall inequality,

$$
|z(t)-x(t)| \leq\left(1+\frac{|b|}{|a+b|}\right) \epsilon \lambda_{\varphi} \varphi(t) E_{m, 1}\left(\frac{K}{1-L} \frac{1}{|\Gamma(\theta)|} \Gamma(m) T^{m}\right), \quad t \in J .
$$

Thus, the problem (0.1) is generalized Ulam-Hyers-Rassias stable.

\section{REFERENCES}

[1] C. Alsina, R. Ger, On some inequalities and stability results related to the exponential function, J. Inequal. Appl., 2, (1998), 373-380.

[2] R. Andriambololona, R. Tokiniaina, H. Rakotoson, Definitions of complex order integrals and complex order derivatives using operator approach, International Journal of Latest Research in Science and Technology, 1(4), (2012), 317-323.

[3] S. Andras, J. J. Kolumban, On the Ulam-Hyers stability of first order differential systems with nonlocal initial conditions, Nonlinear Analysis, 82,(2013),1-11.

[4] A.Arara, M.Benchohra, N.Hamidi, J.J.Nieto, Fractional order differential equations on an unbounded domain, Nonlinear Analysis , 72 (2), (2010), 580-586.

[5] Bertram Ross, Francis H. Northover, A use for a derivative of complex order in the fractional calculus, 9(4),(1977), 400-406.

[6] Z. Bai, H. Lu, Positive solutions for boundary value problem of nonlinear fractional differential equation, Journal of Mathematical Analysis and Applications , 311 (2), (2005), 495-505.

[7] Z. Bai, On positive solutions of a nonlocal fractional boundary value problem, Nonlinear Analysis, 72 (2), (2010), 916-924.

[8] C.S. Goodrich, Existence of a positive solution to a class of fractional differential equations, Applied Mathematics Letters , 23, (2010), 1050-1055.

[9] Z. Bai, H. Lu, Positive solutions for a boundary value problem of nonlinear fractional differential equations, Journal of Mathematical Analysis and Applications, 311,(2005), 495-505.

[10] M. Benchohra, J. E. Lazreg, Existence and Uniqueness results for nonlinear implicit fractional differential equations with boundary conditions, Romanian Journal of Mathematics and Computer Science, 4, (2014), 60-72.

[11] M. Benchohra, S. Bouriah, Existence and stability results for nonlinear boundary value problem for implicit differential equations of fractional order, Morccan Journal of Pure and Applied Analysis, 1(1), (2015), 22-37.

[12] M. Benchohra, S. Hamani, S. K. Ntouyas, Boundary value problems for differential equations with fractional order, Survey in Mathematics and its Applications, (3), (2008), 1-12.

[13] Carla M. A. Pinto, J. A. Tenreiromachado. Complex order van der Pol oscillator. Nonlinear Dynamics, Springer Verlag, 65 (3), 2010, pp.247-254.

[14] A. Granas, J. Dugundji, Fixed Point Theory, Springer-Verlag, New York, 2003.

[15] R.Hilfer, Application of fractional Calculus in Physics, World Scientific, Singapore, 1999.

[16] DH. Hyers, G. Isac, TM. Rassias, Stability of functional equation in several variables, Vol. 34, Progress in nonlinea differential equations their applications, Boston (MA): Birkhauser; 1998. 
[17] D.H. Hyers, On the stability of the linear functional equation, Proc. Natl. Acad. Sci. , USA 27, (1941), 222-224.

[18] R. W. Ibrahim, Generalized Ulam-Hyers stability for fractional differential equations, International Journal of mathematics,23,(2012),doi:10.1142/S0129167X12500565.

[19] S. M. Jung, Hyers-Ulam stability of linear differential equations of first order, Applied Mathematics Letters, 17,(2004),1135-1140.

[20] A. A. Kilbas, H. M. Srivasta, J. J. Trujillo, Theory and application of fractional differential equations, Elsevier B. V, Netherlands,(2016).

[21] E.R. Love, Fractional derivatives of imaginary order, Journal of the London Mathematical Society, 2(2-3), 241-259, (1971).

[22] P. Muniyappan, S. Rajan, Hyers-Ulam-Rassias stability of fractional differential equation, International Journal of pure and Applied Mathematics, 102, (2015),631-642.

[23] R.L. Magin, Fractional Calculus in Bioengineering, Begell House, 2006.

[24] K.S. Miller, B. Ross, An Introduction to the Fractional Calculus and Fractional Differential Equations, Wiley, New York, 1993.

[25] Moustafa El-Shahed, Positive solutions for boundary value problem of nonlinear fractional differential equation, Abstract and Applied Analysis , 2007 (2007) Article ID 10368, 8 pages.

[26] A. Neamaty, M. Yadollahzadeh, R. Darzi, On fractional differential equation with complex order, Progress in fractional differential equations and Apllications, 1(3), (2015),223-227.

[27] I. Podlubny, Fractional differential equations, Academic Press, San Diego, 1999.

[28] Rabha W. Ibrahim, Ulam stability of boundary value problem, Kragujevac Journal of Mathematics, 37(2), (2013), 287-297.

[29] I. A. Rus, Ualm stabilities of ordinary differential equations in a Banach space, Carpathian Journal Mathematics, 26, (2010), 103-107.

[30] S. G. Samko, A.A. Kilbas O. I. Marichev, Fractional Integrals and Derivatives-Theory and Applications, Gordon and Breach Science Publishers, Amsterdam , 1993.

[31] Teodor M. Atanackovi, Sanja Konjik, Stevan Pilipovic, Dusan Zorica, Complex order fractional derivatives in viscoelasticity, Mech Time-Depend Mater, 1, (2016), 1-21.

[32] S.M. Ulam, A Collection of Mathematical Problems, Interscience, New York, 1960.

[33] D. Vivek, K. Kanagarajan, S. Harikrishnan, Dynamics and stability results for fractional integrodifferential equations with complex order, Discontinuity, Nonlinearity and Complexity, (2007), (Accepted manuscript, id: DNC-D-2017-0007).

[34] J. Wang, L. Lv, Y. Zhou, Ulam stability and data dependence for fractional differential equations with Caputo derivative,Electronic Journal of Qualitative Theory of Differential Equations,63,(2011),110.

[35] J. Wang, Y. Zhou, New concepts and results in stability of fractional differential equations,Communications on Nonlinear Science and Numerical Simulations,17,(2012),2530-2538.

[36] S. Zhang, Existence of solution for a boundary value problem of fractional order, Acta Mathematica Scientia. Series B. English Edition , 26 (2), (2006), 220-228.

(D. ViVeK) Department of Mathematics, PSG College of Arts \& Science, Coimbatore-641 014, INDIA.

Email address, D. VIVEK: peppyvivek@gmail.com

(E. M. ELSAYED) Department of Mathematics, Faculty of Science, King Abdulaziz UniverSiTY, JEDDAH 21589, SAUdi ARABIA.

Email address, E. M. ELSAYED: emmelsayed@yahoo.com

(K. KANAGARAjAN) Department of Mathematics, SRi Ramakrishna Mission Vidyalaya College of Arts and Science, Coimbatore-641020, India.

Email address, K. KANAGARAJAN: kanagarajank@gmail.com 